\title{
Induction of Apoptosis in Cultured Rat Gastric Epithelial Cells by Ipriflavone: Comparison with Indomethacin
}

\author{
Satoru TANI, ${ }^{*, a, b}$ Kazuki MATSUdA, ${ }^{a}$ and Toru TANAKA ${ }^{a}$ \\ ${ }^{a}$ Faculty of Pharmaceutical Sciences, Josai University; and ${ }^{b}$ Life Science Research Center, Josai University; $1-1$ Keyaki- \\ dai, Sakado, Saitama 350-0295, Japan. $\quad$ Received November 13, 2003; accepted February 16, 2004
}

Based on a previous report illustrating severe gastrointestinal side effects with ipriflavone, we examined the effects of ipriflavone on cell death in cultured rat gastric epithelial cells compared with other chemicals, including indomethacin. Low concentrations of ipriflavone, indomethacin, dexamethasone, and estradiol all induced cell death in gastric epithelial cells. DNA from cultured cells treated with ipriflavone showed fragmentation by electrophoresis. Also, some of the cultured cells treated with ipriflavone were positively stained by TUNEL. In order to confirm induction of apoptosis by ipriflavone, rescue from ipriflavone-induced cell death with Z-DEVDFMK (0.02-0.1 mM), which is a caspase 3 inhibitor, or PGE $_{2}(0.01-10 \mathrm{~mm})$, was tested. Only Z-DEVD-FMK was observed to rescue the cells from cell death. Similar results were obtained with indomethacin, dexamethasone and estradiol. These results suggest that ipriflavone, indomethacin, dexamethasone and estradiol induce cell death of cultured rat gastric epithelial cells by apoptosis.

Key words gastric epithelial cell; apoptosis; ipriflavone; indomethacin; DNA fragmentation; caspase 3 inhibitor

Adverse reactions can negate or diminish the positive effects of therapeutic agents. Gastric epithelial cells are directly exposed to orally administered drugs. As well, indirect exposure occurs following intestinal absorption via the blood. Thus, it is important to investigate the relationship between gastric epithelial exposure to therapeutic agents and gastrointestinal side effects. Non-steroidal anti-inflammatory drugs (NSAIDs) are an enormously important cause of gastrointestinal lesions. NSAIDs are often used as first line therapy in the treatment of chronic diseases like rheumatoid arthritis. The adverse effects of NSAIDs have been explained, in part, by their mechanism of cyclooxygenase (COX) inhibition, which also inhibits the synthesis of cyto-protective prostaglandin $\mathrm{E}_{2}\left(\mathrm{PGE}_{2}\right)$. Recently, COX-2 selective inhibitors and derivatives of cyto-protective prostaglandins have been developed in an effort to avoid the gastrointestinal side effects observed with NSAIDs. However, indomethacin, which is a NSAID, has also been observed to induce cell death via apoptosis of gastric epithelial cells. ${ }^{1)}$ The mechanism of indomethacin-induced apoptosis is not yet clear, however, a number of factors such as induction of inducible nitric oxide synthase and interleukin- $8,{ }^{2)} \mathrm{p} 53$ and/or c-myc, ${ }^{3)}$ as well as tumor necrosis factor- $\alpha,{ }^{4)}$ are thought to be involved. The gastrointestinal side effects of indomethacin may due to a synergistic effect of COX inhibition and apoptosis. On the other hand, gastrointestinal side effects have been observed with agents other than NSAIDs. Serious gastrointestinal side effects have recently been reported with ipriflavone. ${ }^{5)}$ Ipriflavone is used in the treatment of osteoporosis. It has been shown to inhibit bone loss in combination with estrogen in oophorectomized women. ${ }^{6)}$ However, the mechanism by which ipriflavone induces gastric ulceration is unknown. Thus, we investigated the action of ipriflavone on cultured gastric epithelial cells, compared with indomethacin, dexamethasone and estradiol.

\section{MATERIALS AND METHODS}

Materials Indomethacin, ipriflavone, dexamethasone, estradiol, and ibuprofen were purchased from Wako Pure
Chemical (Tokyo, Japan). Etodolac was generously donated by Japan Wise Redary (Tokyo, Japan). Fetal bovine serum (FBS) and Z-DEVD-FMK, a caspase 3 inhibitor, were purchased from Biofluid Inc. (Rockville, MD, U.S.A.) and Calbiochem-Novabiochem Co. (California, U.S.A.), respectively. Dispase $I^{\circledR}$ and 3-(4,5-dimethylthiazol-2-yl)-2,5-diphenyltetrazolium bromide (MTT) were from Godo Shusei Co. (Tokyo, Japan) and Acros Organics (Belgium, U.S.A.), respectively. All other regents were of the best commercial quality available.

Preparation and Culture of Rat Gastric Epithelial Cells Rat gastric epithelial cells were obtained from Wistar rats weighing about $300 \mathrm{~g}$, using a previously established method. ${ }^{7)}$ Epithelial cells isolated using Dispase from an excised and reversed stomach, were collected by centrifugation at $50 \times \boldsymbol{g}$ for $3 \mathrm{~min}$. After this, the cells were washed twice with culture medium (Dulbecco's Modified Eagle Medium:Ham F-12=1:1) containing gentamicin sulfate $(100 \mu \mathrm{g} / \mathrm{ml})$ by centrifugation. Then, the cells were cultured in collagen (type-I, pig)-coated plastic dishes (Iwaki Glass, Tokyo, Japan) with the same culture medium containing $10 \%$ FBS at $37^{\circ} \mathrm{C}$ in a $\mathrm{CO}_{2}$ incubator. The number of inoculated cells was often observed to influence the results of the MTT assay. Confluent cell layers showed marked resistance against the effects of the chemicals. The cells (about $1 \times 10^{6}$ cells) were inoculated into $35 \mathrm{~mm} \phi$ culture dishes so as to form numerous small islets.

Measurement of Cell Viability Cell viability was measured with MTT assays 1 and $48 \mathrm{~h}$ after the addition of each test chemical. In this paper, MTT assays were used to identify the primary cause of cell death at different points during the incubation period, that is, the primary cause of acute cell death within the first hour, as well as that occurring after $48 \mathrm{~h}$.

Extraction of DNA and Electrophoresis Extraction of DNA from cultured cells was carried out using a phenolchloroform mixture. ${ }^{8)}$ Extracted DNA was separated by electrophoresis on a $2 \%$ agarose gel. After electrophoresis, the pattern of fluoresced DNA following exposure to ethidium bromide was photocopied. 
Stains Cultured cells were stained with periodic acid Schiff's stain (PAS), paradoxical concanavalin A stain (PCS), ${ }^{9)}$ and the TUNEL stain (DeadEnd colorimetric TUNEL System, Promega Co., Madison, WI, U.S.A.). ${ }^{10)}$
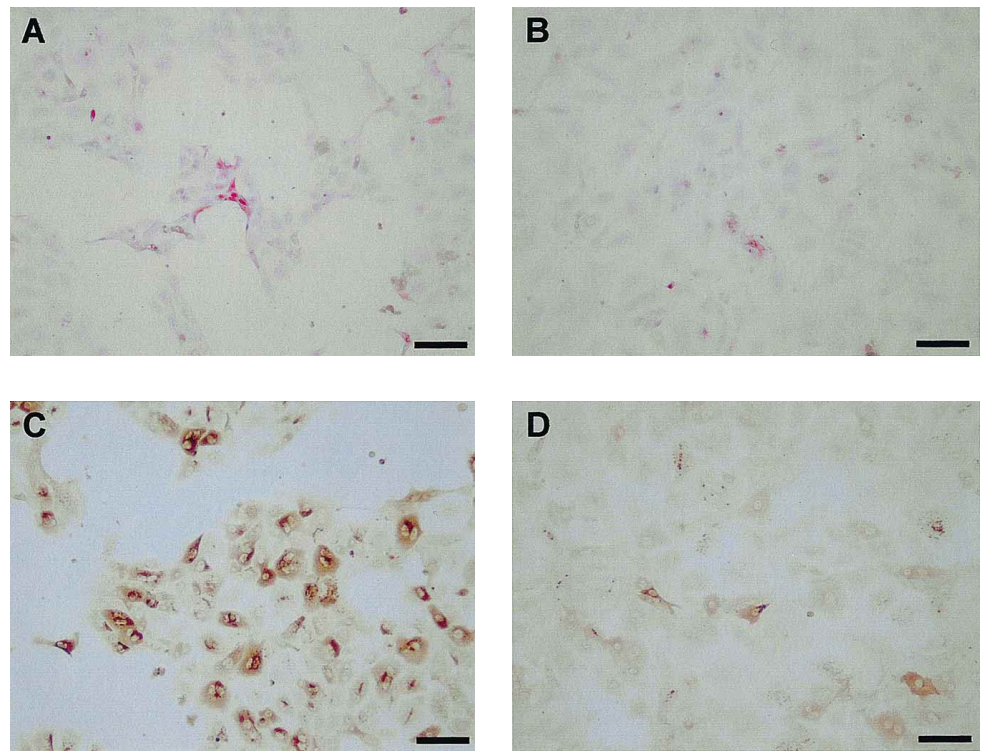

Fig. 1. Photomicrographs of Cultured Rat Gastric Epithelial Cells Stained with PAS and PCS

Cultured cells were stained by PAS (A, B) and PCS (C, D), 2 (A, C) and 4 (B, D) days after inoculation. The bar shown in each photo represents $100 \mu \mathrm{m}$.
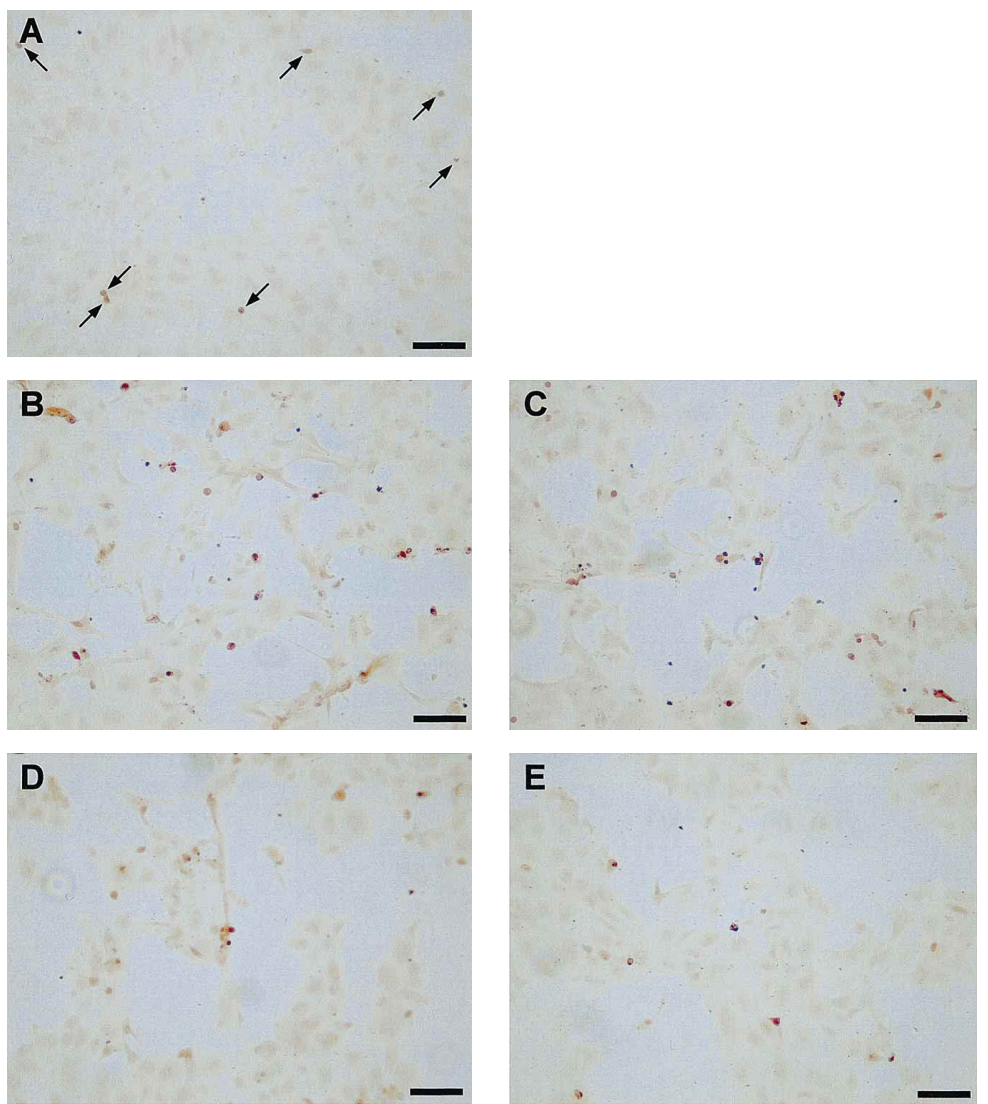

Fig. 4. Photomicrographs of TUNEL-Stained Cells in Culture after Treatment

Cells were stained with TUNEL $16 \mathrm{~h}$ after treatment with A) the vehicle (DMSO 0.1\%), B) indomethacin $(0.6 \mathrm{~mm}), \mathrm{C})$ ipriflavone $(0.018 \mathrm{~mm})$, D) dexamethasone $(0.85 \mathrm{~mm})$, and E) estradiol $(0.05 \mathrm{~mm})$. The bar shown in each photo represents $100 \mu \mathrm{m}$. The arrows in A) indicate TUNEL-positive cells (arrows are not included in the other photos). 

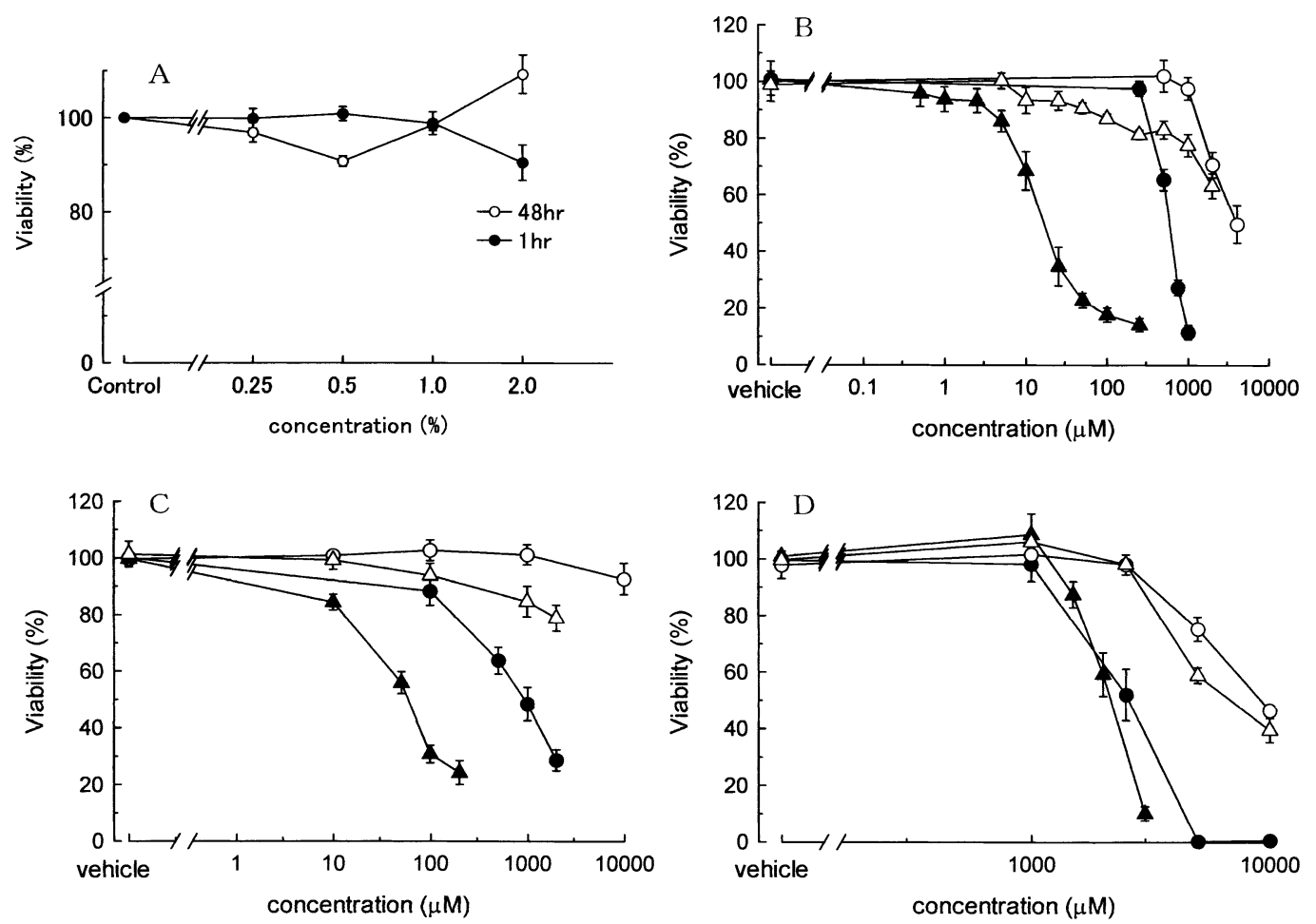

Fig. 2. Effects of Indomethacin, Ipriflavone and Related Chemicals on the Viability of Cultured Rat Gastric Epithelial Cells

Dose-response curves examining the effects of A) the vehicle (DMSO), B) indomethacin $(\boldsymbol{\bullet}, \bigcirc)$ and ipriflavone $(\boldsymbol{\Delta}, \triangle), \mathrm{C})$ dexamethasone $(\boldsymbol{\bullet}, \bigcirc)$ and estradiol $(\boldsymbol{\Delta}, \triangle)$, and D) ibuprofen $(\boldsymbol{O}, \bigcirc)$ and etodolac $(\boldsymbol{\Delta}, \triangle)$, on cell viability were made based on the results of the MTT assays. Each data point represents the mean \pm S.E. of 3 to 6 separate experiments. The closed and open symbols represent cell death 48 and $1 \mathrm{~h}$ after the addition of each chemical, respectively.

were stained with PAS and PCS, respectively. The cultured cells were thought to be mucus cells, mainly mucus neck cells, since they were both PAS- and PCS-positive (Fig. 1). However, the intensity of staining decreased as the cells were further passaged.

Chemical Effects on Cell Viability Cell viability was measured by MTT assay 1 and $48 \mathrm{~h}$ after the addition of indomethacin, ipriflavone, dexamethasone, estradiol, etodolac, ibuprofen, and DMSO, which was used as a vehicle (Fig. 2). Generally speaking, lower concentrations of the various chemicals induced cell death at $48 \mathrm{~h}$, compared to the concentrations required for cell death at $1 \mathrm{~h}$. Drug concentrations resulting in a $50 \%$ decline in cell viability at 1 and $48 \mathrm{~h}$ were designated acute and delayed $\mathrm{CC}_{50}$ concentrations, respectively. The acute $\mathrm{CC}_{50}$ of indomethacin was about $4 \mathrm{~mm}$, and the delayed $\mathrm{CC}_{50}$ was about $0.5 \mathrm{~mm}$. On the other hand, the acute $\mathrm{CC}_{50}$ of ipriflavone was about $0.05 \mathrm{~mm}$, and the delayed $\mathrm{CC}_{50}$ was about $0.01 \mathrm{~mm}$. The delayed $\mathrm{CC}_{50}$ values of dexamethasone and estradiol were 1 and $0.05 \mathrm{~mm}$, respectively. Dexamethasone and estradiol were not acutely cytotoxic to cultured gastric epithelial cells, even at concentrations of 10 and $1 \mathrm{~mm}$, respectively. The acute $\mathrm{CC}_{50}$ values of ibuprofen and etodolac were about $10 \mathrm{~mm}$, while their delayed $\mathrm{CC}_{50}$ values were approximately $2 \mathrm{~mm}$. The dose-response curve of cell death following exposure to indomethacin differed from the dose-response curves of other agents. The acute and delayed $\mathrm{CC}_{50}$ values of indomethacin were similar and indomethacin had a steep dose-response curve. DMSO, which was used as the vehicle, was not cytotoxic at any of the concentrations examined.

DNA Fragmentation In order to identify the cause of cell death, chemical-induced DNA fragmentation was exam-

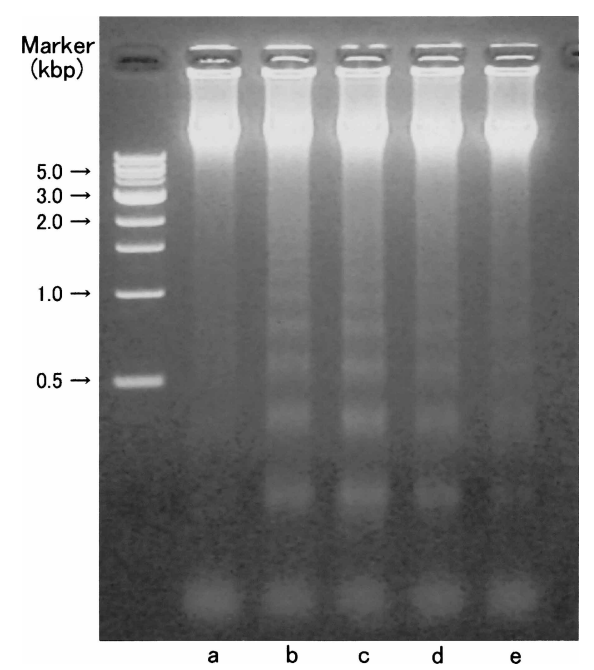

Fig. 3. Chemical-Induced Apoptotic DNA Fragmentation of Chromosomal DNA as Determined by Electrophoresis on a 2\% Agarose Gel

a: vehicle (DMSO, $0.1 \%)$, b: indomethacin $(0.6 \mathrm{~mm})$, c: ipriflavone $(0.018 \mathrm{~mm}), \mathrm{d}$ : dexamethasone $(0.85 \mathrm{~mm})$, e: estradiol $(0.05 \mathrm{~mm})$.

ined by electrophoresis. Indomethacin, ipriflavone, dexamethasone and estradiol were selected among the tested chemicals. The DNA obtained from cultured cells after $48 \mathrm{~h}$ was completely destroyed, showing a smear pattern on electrophoresis. Examination of the electrophoresis patterns of DNA after different intervals of culture revealed ladder patterns of DNA after $16 \mathrm{~h}$ of incubation with each chemical (Fig. 3). DNA fragmentation was observed in cells treated with ipriflavone, indomethacin, dexamethasone and estradiol, while minimal fragmentation of DNA was observed in cells 
Table 1. Effects of $\mathrm{PGE}_{2}$ on Indomethacin-, Ipriflavone-, Dexamethasoneand Estradiol-Induced Cell Death at $48 \mathrm{~h}$

\begin{tabular}{lccccc}
\hline \hline \multicolumn{1}{c}{$\mathrm{PGE}_{2}$} & $0:$ control & $0.01 \mathrm{~mm}$ & $0.1 \mathrm{~mm}$ & $1.0 \mathrm{mM}$ & $10 \mathrm{mM}$ \\
\hline Indomethacin & $56.6 \pm 2.7$ & $54.8 \pm 4.5$ & $59.3 \pm 4.2$ & $59.5 \pm 4.6$ & $58.7 \pm 4.4$ \\
Ipriflavone & $42.2 \pm 2.9$ & $47.6 \pm 2.8$ & $49.9 \pm 2.0$ & $49.6 \pm 2.3$ & $45.1 \pm 2.9$ \\
Dexamethasone & $54.7 \pm 0.6$ & $58.6 \pm 1.8$ & $56.4 \pm 3.3$ & $58.7 \pm 2.3$ & $61.5 \pm 3.9$ \\
Estradiol & $70.5 \pm 3.5$ & $71.2 \pm 4.2$ & $69.4 \pm 4.5$ & $70.0 \pm 3.9$ & $70.6 \pm 2.8$ \\
\hline
\end{tabular}

Cells were cultured for $48 \mathrm{~h}$ with or without the indicated concentrations of $\mathrm{PGE}_{2}$ and ipriflavone $(0.018 \mathrm{~mm})$, indomethacin $(0.6 \mathrm{~mm})$, dexamethasone $(0.85 \mathrm{~mm})$, estradiol $(0.05 \mathrm{~mm})$, or DMSO $(0.1 \%)$. After this, the proportion of viable cells within each culture was measured with a MTT assay. Results are expressed as a percentage of the proportion of viable control cells observed after exposure to DMSO alone. Each value represents the mean \pm S.E. of 4 separate experiments.

Table 2. Effects of Z-DEVD-FMK on Indomethacin-, Ipriflavone-, Dexamethasone- and Estradiol-Induced Cell Death at $48 \mathrm{~h}$

\begin{tabular}{lclcc}
\hline \hline Z-DEVD-FMK & 0 : control & $0.02 \mathrm{~mm}$ & $0.05 \mathrm{~mm}$ & $0.1 \mathrm{~mm}$ \\
\hline Indomethacin & $63.8 \pm 2.0$ & $99.7 \pm 1.3^{* *}$ & $113.1 \pm 2.3 * *$ & $99.8 \pm 4.2^{* *}$ \\
Ipriflavone & $49.1 \pm 7.3$ & $65.6 \pm 6.0$ & $83.7 \pm 6.2 *$ & $84.3 \pm 3.0^{*}$ \\
Dexamethasone & $37.9 \pm 4.0$ & $48.1 \pm 5.1$ & $56.3 \pm 5.8$ & $83.8 \pm 6.5^{* *}$ \\
Estradiol & $61.8 \pm 3.9$ & $67.6 \pm 2.8$ & $71.9 \pm 4.4$ & $91.0 \pm 2.7 * *$ \\
\hline
\end{tabular}

Cells were cultured for $48 \mathrm{~h}$ with or without the indicated concentrations of $\mathrm{Z}$ DEVD-FMK and ipriflavone $(0.018 \mathrm{~mm})$, indomethacin $(0.6 \mathrm{~mm})$, dexamethasone $(0.85 \mathrm{~mm})$, or estradiol $(0.05 \mathrm{~mm})$, after which viable cells were measured with a MTT assay. Results are expressed as a percentage of the proportion of viable control cells observed after exposure to $0.1 \%$ DMSO alone. Each value represents the mean \pm S.E. of 4 or 3 experiments examining dexamethasone or one of the other drugs, respectively. $* p<0.01 ; * * p<0.001$ compared with the control value.

treated with DMSO, as a control.

TUNEL Stain After $16 \mathrm{~h}$ of treatment with the delayed $\mathrm{CC}_{50}$ concentration of each chemical, the cells were stained by TUNEL. An increased number of TUNEL-positive cells were detected, compared with control cells, however, a few cells had detached from the culture dish following addition of the chemicals, as seen in the micrograph (Fig. 4).

Effects of PGE $_{2}$ and Z-DEVD-FMK The effects of $\mathrm{PGE}_{2}$ and Z-DEVD-FMK on cell death induced by ipriflavone, indomethacin, dexamethasone and estradiol at their delayed $\mathrm{CC}_{50}$ concentrations were examined. $\mathrm{PGE}_{2}$ did not protect against indomethacin- and ipriflavone-induced cell death, even at high concentrations (Table 1). However, rescue from cell death following exposure to ipriflavone, indomethacin, dexamethasone and estradiol was achieved with ZDEVD-FMK. These results were significant and dosedependent. In particular, marked protection against indomethacin-induced cell death was observed (Table 2).

\section{DISCUSSION}

We measured the cell death of cultured rat gastric epithelial cells with MTT assays 1 and $48 \mathrm{~h}$ after the addition of various therapeutic agents. It is thought that those agents which induced cell death via necrosis within $1 \mathrm{~h}$ of treatment likely had non-specific cytotoxic activity. Induction of apoptosis by a therapeutic agent resulted in cell death after a longer period of incubation. It is not surprising that chemicals which demonstrate toxic effects induce ulceration over a period of time. A number of therapeutic agents are known to have the gastrointestinal side effects. In this paper, we demonstrated that cell death induced by ipriflavone, NSAIDs, dexamethasone, and estradiol is due to apoptosis, based on our examination of DNA fragmentation and the TUNEL assay results. Since the cultured cells were often observed to detach from the culture dish following treatment, it was also necessary to check the viability of the detached cells. More than $75 \%$ of detached and floating cells collected by gentle centrifugation were not viable (data not shown). It was therefore thought that detachment from the dish was often secondary to cell death.

Among the NSAIDs examined in this paper, indomethacin was the strongest inductor of apoptosis. A comparison of ibuprofen and etodolac revealed that etodolac is able to induce apoptosis at much lower concentrations than ibuprofen. This is consistent with the observation that etodolac has milder gastrointestinal side effects. As shown in this paper, indomethacin-induced apoptosis was rescued by Z-DEVDFMK, but not $\mathrm{PGE}_{2}$. These results suggest that NSAID-induced apoptosis is not directly related to COX inhibition. A1though $\mathrm{PGE}_{2}$ is the main product of COX activity within the gastric mucosa, it is possible that other prostaglandins also protect against ulceration. NSAID-induced apoptosis might be associated with the anti-inflammatory pathway, since dexamethasone is also a strong inductor of apoptosis.

There are known differences in gastric secretion among males and females. In this paper, we used gastric mucosal cells from male rats. Since gastric epithelial cells from female rats have been shown to possess estrogen receptors, ${ }^{11)}$ it is thought that male rats might share similar receptors. Ipriflavone was observed to induce apoptosis at low concentrations. Estradiol also induced apoptosis, but at a much greater delayed $\mathrm{CC}_{50}$ concentration than ipriflavone. Therefore, ipriflavone-induced apoptosis is not directly associated with estrogen activity. These results suggest that the gastrointestinal side effects of ipriflavone are due to apoptosis. Usually estrogen stimulates cell proliferation within female reproductive organs. With respect to estrogen-induced apoptosis, different sensitivities are observed among different cell lines. Tamoxifen, an anti-estrogen, has been observed to induce apoptosis of breast cancer cells, ${ }^{12)}$ while estradiol has been observed to induce lymphocyte apoptosis. ${ }^{13)}$ Thus, it is thought that the estrogen receptor is closely linked with cell growth and apoptosis. A number of biologically active compounds have been identified in flavonoids, including ipriflavone. ${ }^{14)}$ Flavonoids are used as therapeutic agents and are present in a number of dietary supplements. They are widely commercially available and sold due to the fact that they are natural substances. However, it is hoped that the active ingredients of flavonoids can be isolated and concentrated in order to achieve therapeutic effects beyond those observed with dietary supplements. Further studies aimed at elucidating the potential side effects of flavonoids are necessary.

\section{REFERENCES}

1) Tomisato W., Tsutsumi S., Rokutan K., Tsuchiya T., Mizusima T., Am. J. Physiol. (Gastrointest. Liver Physiol.), 281, G1092-G1100 (2001).

2) Imamine S., Akbar F., Mizukami Y., Matsui H., Onji M., Int. J. Path., 82, 221-229 (2001).

3) Zhu G. H., Wong B. C., Ching C. K., Lai K. C., Lam S. K., Biochem. Pharmacol., 58, 193-200 (1999).

4) Fiorucci S., Antonelli E., Morelli A., Dig, Liver Dis., 33, S35-S43 (2001).

5) Matsuoka M., Yoshida Y., Hayakawa K., Fukuchi S., J. Gastroenterol., 33, 267-271 (1998). 
6) Nozaki M., Hashimoto K., Inoue Y., Ogata R., Okuma A., Nakano H., Int. J. Gynaecol. Obstet., 62, 69-75 (1998).

7) Tani S., Okuda M., Morishige R., Tanaka T., Biol. Pharm. Bull., 20, 482-485 (1997).

8) Asako H., Kubes P., Wallace J., Gaginella T., Wolf R. E., Granger D. N., Am. J. Physiol., 262, G903-G908 (1992).

9) Katsuyama T., Spicer S. S., J. Histochem. Cytochem., 13, 211-234 (1978).

10) Gavrieli Y., Sherman Y., Ben-Sasson S. A., J. Cell Biol., 119, $493-$
501 (1992).

11) Campbell-Thompson M., Reyher K. K., Wilkinson L. B., J. Endocrinol., 171, 65-73 (2001).

12) Salami S., Karami-Tehrani F., Clin. Biochem., 36, 247-253 (2003).

13) Azzarolo A. M., Eihausen H., Schechter J., Exp. Eye Res., 77, 347354 (2003).

14) Ren W., Quao Z., Wang H., Zhu L., Zhang L., Med. Res. Rev., 23, $519-534$ (2003). 